\title{
Analysis of Nanostructures by Magneto-optical Images
}

\author{
A. Nedzved ${ }^{a, b}$, W. Dobrogowski ${ }^{b}$, S. Ablameyko ${ }^{a}$, M. Tekielak $^{b}$, and A. Maziewski ${ }^{b}$ \\ ${ }^{a}$ United Institute of Informatics Problems, National Academy of Sciences of Belarus, \\ ul. Shurganova 6, Minsk, 220012 Belarus \\ ${ }^{b}$ Institute of Experimental Physics, University of Bialystok, Lipowa 41, Bialystok, 15-424 Poland \\ e-mail: nedzveda@newman.bas-net.by
}

\begin{abstract}
The analysis of magnetic domain structures using optical and scanning microscopic images is described. These structures are often observed upon thin films being affected by magnetic fields. The described technique is grounded in the separation of objects into four structural types that are the basis of almost all patterns in an image. They are blobs, fronts, needles, and dendrites. The functions for analyzing these basic structures have been developed. For the description and classification of the structures, a set of specific characteristics for each structural type has been determined. A ZUBR system for magneto-optical image processing is briefly described.
\end{abstract}

DOI: $10.1134 / \mathrm{S} 1054661809020175$

\section{INTRODUCTION}

Currently, nanotechnologies are becoming more and more important; consequently, the requirements for the research in this field have increased. One of the directions that is actively being introduced into technological processes is the study of the magneto-optical properties of ultrathin films.

In recent years, optical microscopy has been a powerful tool for studying the characteristics of magnetic fields, in particular, in ultrathin magnetic films. Modern optical microscopes operate with spatial resolution on a micron scale and with time intervals less of than a millisecond, which makes it possible to study static distributions of magnetic fields, penetration into magnetic materials, residual magnetization, magnetic field propagation fronts, etc.

An important application of optical microscopy is the determination of the local distributions of magnetization in ultrathin magnetic fields. These distributions can be obtained from a fully saturated sample in both opposite directions [1].

Automation of the analysis of magneto-optical images requires the undivided attention and high accuracy of the experimentalist. Therefore, this field has been developing for a fairly long time. It is the most popular to use for the analysis general-purpose software for obtaining and processing images of high resolution and large brightness depth. These systems include the image analyzers made by Leica (http://www.microscopy-imaging.com/), Kontron Electronic (http://www.zeiss.ru/production/1/micro_prinadl_

Received March 18, 2008 ks/index.htm), Image-Pro Plus (media Cybernetics, http://www.mediacy.com/ippage.htm), Image Warp (http://www.imagewarp.com), etc. The IMAQ package (National Instruments, www.ni.com) is one of the most successful, as it is integrated into a LabView shell providing interaction with a wide variety of physical devices and possessing an interface that can be intuitively comprehended by engineers and physicists. The VinView package (Precision Instruments) is widely used for the interaction with old equipment, as it allows processing very large images and their sequences using weak-resource computer techniques. However, all these systems use traditional tools of image processing and differ from each other by their ways of implementation, operation organization, and image presentation. Unfortunately, these systems are not specialized for operation with thin magnetic films and require a specialist familiar with the system features and image processing.

In the first specialized systems, emphasis was put on the improvement of magneto-optical images $[2,3]$. The attempts to focus on the analysis of the structural features [4] and dynamical peculiarities of images [5] started relatively not long ago.

At present, three types of systems for analyzing and processing magneto-optical images are differentiated; they are general-purpose systems, systems with additional possibilities, and systems for solving specific problems. However, to the best of our knowledge, there are no specialized systems where the specific characteristics of magnetic structures can be analyzed. Therefore, the development of a technique for analyzing magneto-optical structures is relevant. 
(b)

(a)

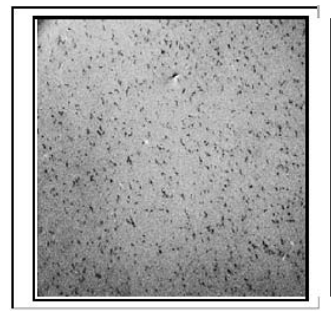

(c)
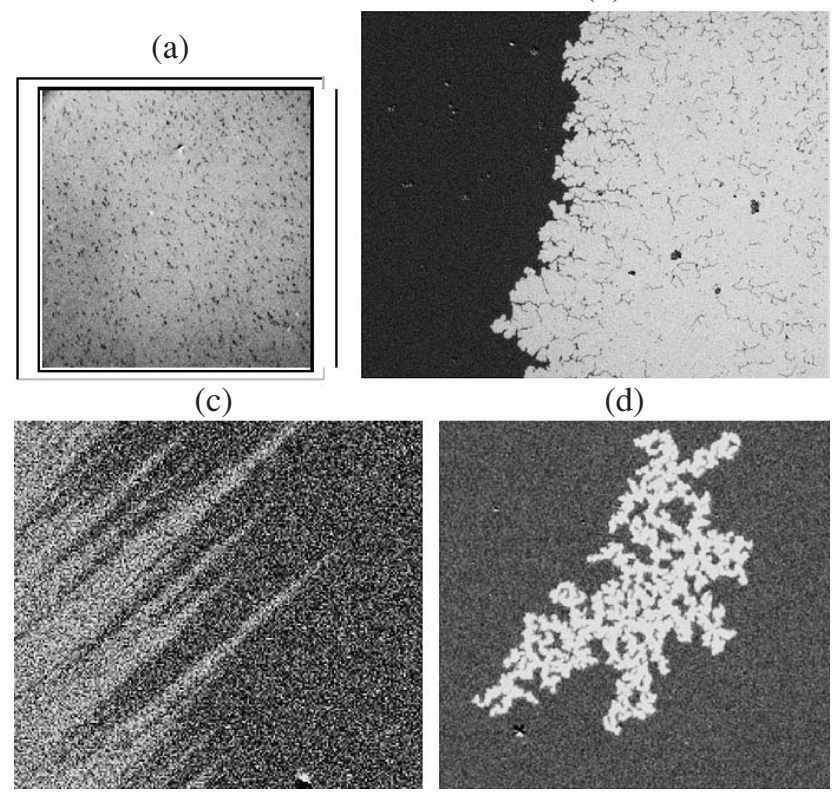

(d)

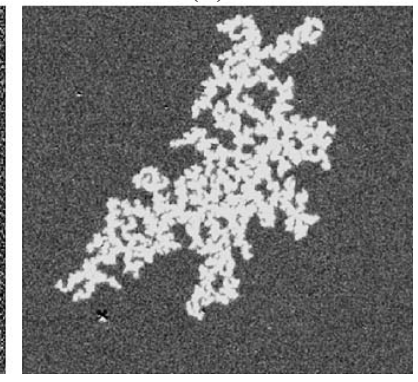

Fig. 1. The four main types of magnetic structures: blobs (a), fronts (b), needlelike structures (c), and dendrite-like structures $(\mathrm{d})$.

\section{BASIC TYPES OF MAGNETIC STRUCTURES}

Under the effect of a magnetic field on different materials, many geometric structures [6-8] can be formed by disoriented domains [7]. However, in our observation the formation of these structures in ultrathin cobalt layer deposited on different buffer layers $(\mathrm{Au}, \mathrm{Ag}, \mathrm{Mo})$, is based on the four configurations that are created in two cases of magnetization reversal of a material.

1. The process of magnetization reversal is characterized by numerous centers of nucleation of magnetic domains in the region under study. With a change of the external magnetic field amplitude, the size of the region around the nucleation center changes forming blobs. The blobs (Fig. 1a) have an arbitrary shape with comparable length and width but no internal structure. In magneto-optical images, they change isotropically with the magnetic field.

2. The process of magnetization reversal is characterized by a limited number of magnetic domain nucleation centers or their absence in the region under study. In this case, complex magnetic structures growing from a certain ground are formed. They can be classified as fronts, needles, and dendrites.

(a) A front (Fig. 1b) is a large extended object that characterizes the boundary of changing magnetic domains and moves in one direction. With a change in the magnetic field, the sizes of the regions change and the front replaces and changes its shape.

(b) The needlelike structure (Fig. 1c) consists of objects elongated in one direction with a relatively smooth boundary that end with a sharp angle (needles). With a change in the magnetic field, the length and width of such objects change; however, there is domination of the needle length growth.

(c) The dendrite-like structure [9] (Fig. 1d) is very complex geometrically and consists of dendrites. Dendrites are objects with a treelike branching structure. This term is of old origin; dendrite shapes were mentioned as early as in 1774 by G. Verner. A dendrite is a branching object that occurs upon accelerated or constrained growth under nonequilibrium conditions when an object splits following certain laws. As a result, the object loses its initial integrity and disordered blocks, branches, or fingers appear. They branch and grow to different sides similar to a tree, and the regularity of the shape of the initial object is lost as the object grows. The process of dendrite formation is called dendrite growth. Many models of dendrite formation have been proposed.

Using the above classification, other forms of magnetic domain structures can be characterized.

\section{FORMATION \\ OF MAGNETO-OPTICAL STRUCTURES}

The typical technique for the magnetic domain structure visualization is the magneto-optical imaging. In the reflection configuration the magnetization spatial distribution analysis based on Kerr effect [7].

The magneto-optic imaging based on the rotation of the plane of polarization of linearly polarized light upon reflection from a magnetic surface. The reflected beam is passed through an analyzer which is nearly crossed with the incident light polarizer (see Fig. 2). As a result, the component of the light that maintained its original polarization state after the interaction with the magnetized surface is attenuated while the component generated through the magneto-optical interaction is allowed to pass to the detector [10]. The domain contrast in the image is directly sensitive to the magnitude and direction of magnetization.

Maximum attenuation of the incident light is achieved when the polarizers are perfectly crossed, however Kerr component of the light is very weak and would require an extremely sensitive detector. By allowing some of the incident light to reach the detector the Kerr signal and the background (incident) signal are allowed to add coherently contributing to a higher signal which is easily registered by conventional detectors.

Standard wide field Kerr microscope, which immediately provide an image of a certain sample areas, are based on commercial reflected light microscopes with strain-free optics to allow for polarization microscopy. The microscope is equipped with special electro-magnets system, Xe lamp and a high sensitivity digital CCD camera. 


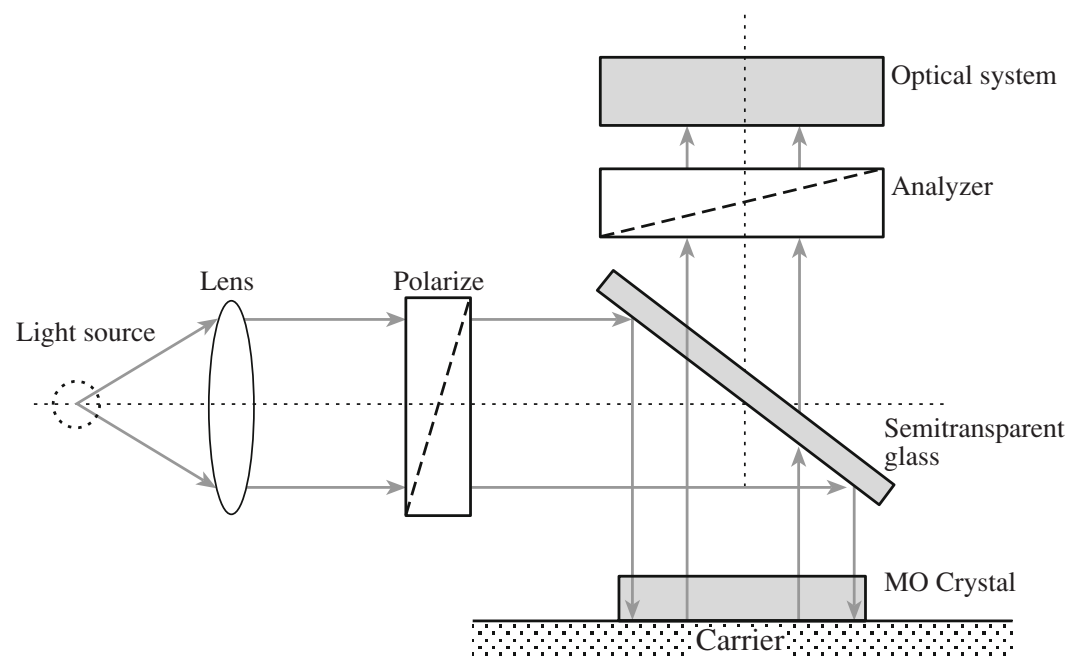

Fig. 2. Polaroid facility for magneto-optical image visualization in microscopy.

The optical flow is registered by a digital camera and processed by a specific algorithm of magneto-optical contrast to enhance the signal-to-noise ratio and contrast the image. There are two types of the magnetooptical contrast: with a constant field and with an alternating field. In order to obtain the magneto-optical contrast at a constant field, several images are captured with different positions of the analyzer, and the component constant relative to the magnetization and an additional (magneto-optical) component corresponding to the effective Kerr amplitude are selected.

In the general case, when the analyzer is rotated towards the polarizer by an angle of $\alpha$, the transformed brightness in the system of the magneto-optical microscopy changes according to the following law:

$$
I=I_{0} \sin ^{2}(\alpha-\theta),
$$

where $\theta$ is the change in the polarization angle for the transmitted light, and $I_{0}$ is the light source brightness.

As a result, the image consists of two components: that constant in all the images and the magneto-optical in the images for the rotated analyzer. The constant component is obtained when the polarizer and the analyzer are positioned at the same angle; otherwise, the magneto-optical component is obtained.

If the constant component of the image is considered to be the background, the image of the magnetooptical contrast can be obtained using a traditional operation of background correction [11]:

$$
C_{x, y}=\frac{\left(I_{x, y}-B_{x, y}\right)\left(W_{\max }-B_{x, y}\right)}{\left(W_{x, y}-B_{x, y}\right)}
$$

where $I_{x, y}$ is the pixel brightness of the image with the magneto-optical component; $B_{x, y}$ is that of the image with the constant component; $W_{x, y}$ is that of the image at the complete saturation of a CCD camera (flash); $W_{\max }$ is the maximum brightness of the image at the camera saturation; and $C_{x, y}$ is the new pixel brightness in the corrected image.

With no regard for the camera saturation, this formula can be considerably simplified and the image of the magneto-optical signal is the difference between these two components. In order to simplify the further processing, it is effective to use the modulus of the difference:

$$
I=\left|I_{n}-I_{k}\right|,
$$

where $I$ is the contrasted image, $I_{n}$ is the image of the constant component, and $I_{k}$ is the image of the magneto-optical component.

In the case of the alternating field, the resulting image is obtained on the basis of the difference between the images obtained without the field (Fig. 1a) and with the field (Fig. 1b):

$$
I=\left|I_{d s}-I_{f}\right|,
$$

where $I$ is the contrasted image, $I_{d s}$ is the image made without the field, and $I_{f}$ is the image obtained with the field.

However, the brightness of the magneto-optical component is small and obtaining such images with digital cameras requires their operation at the breaking point. Therefore, the magneto-optical images are accompanied by a strong speckled background. This problem is partially solved by increasing the exposure time, but the latter is limited by the performance capabilities of the camera. Therefore, during the image input, the "accumulation" technique is used. The resulting image is determined as the sum of the images whose number is obtained during the experiment.

Thus, obtaining a magneto-optical image requires selection of the polarizer and analyzer angles, the accu- 

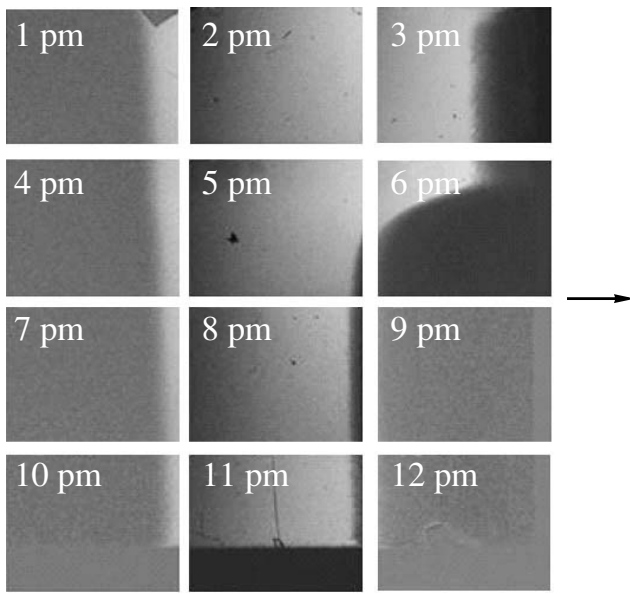

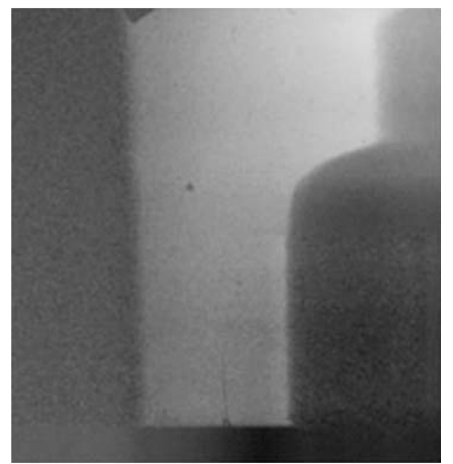

Fig. 3. Stitching with brightness smoothing on the boundaries. mulation time, the number of images in the accumulation procedure, and the values of the magnetic field at which the domain structure is studied.

However, the obtained image of the magnetization can be considerably distorted due to the instability of the light source, the mechanical drift of the sample, physical defects, and other factors. The use of the methods of analyzing and processing the images allows partial correction and improvement of the quality of the images, which makes it possible to study geometrically complex nanostructures formed in materials under various physical conditions.

\section{MAGNETO-OPTICAL IMAGE INPUT AND PREPROCESSING}

The images can be input by a CCD camera or by reading files. Most frequently, images of 8 and 16 bits/pixel are used; however, there exist complexes that allow obtaining denser (32 bits/pixel) images and the real presentation formats.

Automatic stitching of the magneto-optical images is extremely complicated for some reasons that do not allow a correlation function to find the cross points of the images [12]. These reasons are the following:

- The strong speckled background causes a large dispersion of the densimetric characteristics.

- There are no strong brightness drops and objects influencing the maximum of the correlation function.

- The long image capture time and the complex optical path cause noncoincidence of the brightness in the cross and boundary regions.

-In the break between image inputs, the domain structure of the sample can change, which can result in the coexistence of different structures in the region of the image transfer.

The image cross zone is obtained by the position of the maxima after autocorrelation of the target fragments [13]. The result of this operation is illustrated in
Fig. 3. However, in the complex case, when, in the breaks between capturing images, the domain structure changes, the proposed stitching method yields a priori incorrect results. Therefore, the cross coordinates should be searched for using images without structures, i.e., background images. These images can be obtained at zero angle between the analyzer and the polarizer in the absence of a magnetic field without changing the region of interest. Thus, by processing the background image, one can obtain the coordinates for assembling and use them for the images with structures.

After the cross region is found, the brightness line is aligned along the stitching line. This operation is based on building the brightness profile along the stitching line for each image averaged by the stitching direction. The difference between the profiles corresponds to the brightness shift for each image line. However, if the defects fall in the stitching line, they cause traces on the image under correction. In order to eliminate this problem, the brightness profile is processed by an averaging filter [13], which corrects the distortions caused by possible defects.

\section{MAGNETO-OPTICAL IMAGE PREPROCESSING}

Specific features of the magneto-optical images require special preprocessing and improvement of the initial sample. In virtue of the variety of problems to be solved, it is difficult to determine the preprocessing sequence. However, it will generally be as is shown in Fig. 4. The scheme is based on the cycle of accumulation of image fragments from which the complete image of the magneto-optical sample is formed by stitching. Prior to stitching, the operations of background correction and morphological and low-frequency filtering are accomplished in order to improve the optical characteristics of an image and eliminate physical noise. The background correction requires the background image and the sample image. In the case 


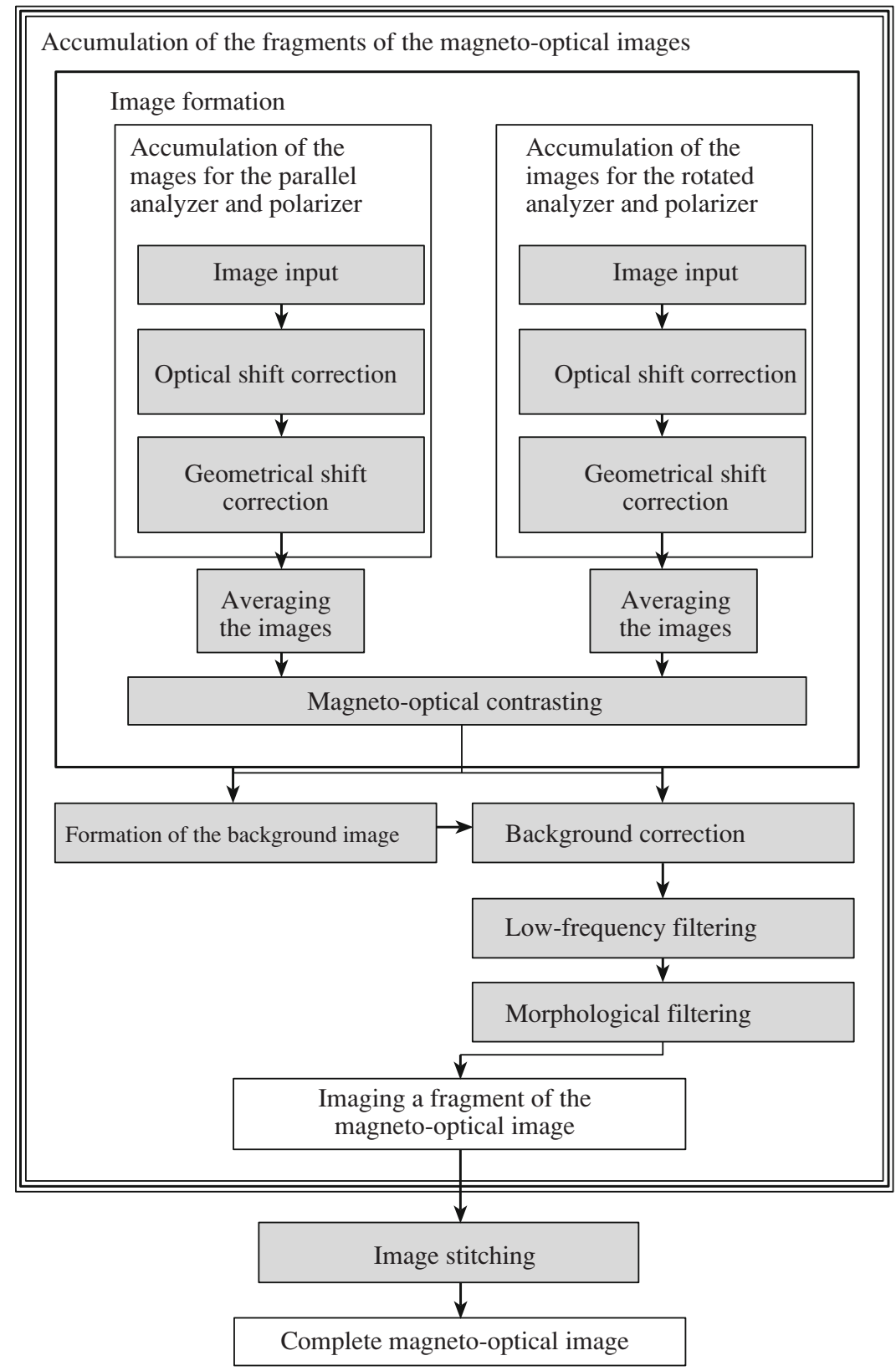

Fig. 4. Generalized scheme for the improvement of magneto-optical images.

when the background image cannot be obtained directly by the magneto-optical methods, it is formed by averaging images.

The proposed preprocessing scheme (Fig. 4) allows correction of the distortions and defects occurring during the formation of the magneto-optical images in order to prepare the latter for the high-level operations of selection and recognition of the magnetic nanostructures. Here, we list the most frequent distortions and defects in magneto-optical microscopy that can be considerably reduced by the image preprocessing.
-Geometrical shift. During the long image capture time, the geometrical shift of a sample occurs, which causes false objects and defects upon magneto-optical contrasting.

-Optical shift. During capturing the image, the brightness characteristics change due to the instability of the light sources.

- Residual noise. The technical limitations of the input system and the exposure time do not eliminate all the noise.

- Changes in the light parameters. The rotation of the analyzer causes different light parameters for differ- 


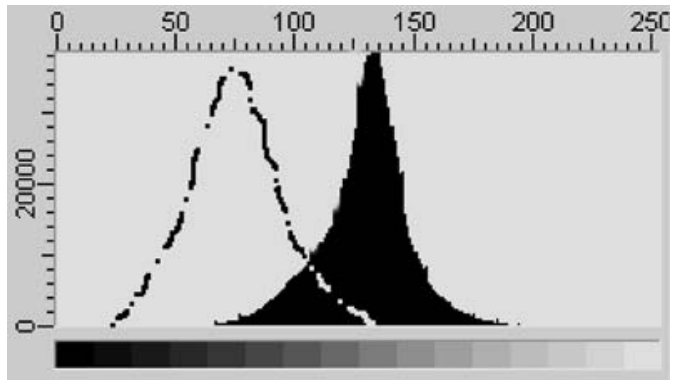

Fig. 5. Histogram shift upon optical drift. The brightness histogram before optical drift is shown by a dashed line.

ent angles of polarization; as a result, an individual background appears for each angle.

- Limited region of interest. The powerful enlarging and the resolution of the microscope often do not allow obtaining the entire object.

The correction of each type of defect requires a separate stage of processing and obtaining the image that includes specific functions.

\subsection{Geometrical Shift}

In order to determine the parameters of the geometrical shift, it is necessary to find the objects in the image sequence that do not change with the applied field and angle of polarization. These objects are physical defects. The selection is based on threshold segmentation, which results in a sequence of binary images. Their conjunction with the previous ones leads to the determination of the constant image elements corresponding to the fragments of the physical defects. These fragments correspond to the cross region of the defect before and after the shift; therefore, they allow the accurate determination of the shifted defect in the next image. The defects are determined by a coupled-component approach, in which the initialization points are the cross regions of the shifted and unshifted defects. The characteristics of the shift are determined via the coordinates of the center of mass, which are calculated using the following formula [14]:

$$
x_{c}=\frac{\sum_{i=0}^{n} x_{i}}{n}, \quad y_{c}=\frac{\sum_{i=0}^{n} y_{i}}{n},
$$

where $x_{i}$ and $y_{i}$ are the coordinates of an object pixel, and $n$ is the number of pixels in the object. The geometrical shift is determined as the difference of the coordinates of the image center of mass. After the determination of the shift, the images are shifted in the initial sequence.

\subsection{Optical Drift}

Instability of the light sources upon capturing the image sequence causes the shift and distortion of the brightness histogram (Fig. 5). Smoothing and scaling the histogram by the characteristics of the first image in the sequence makes it possible to partially compensate for this defect.

The histogram shift is determined from the difference of the mean values of the histograms:

$$
\text { shift }=\frac{\sum_{i m g 1} x-\sum_{i m g 2} x}{n},
$$

where $i m g 1$ is the index of the first image, img 2 is the index of the other image, $x$ is the brightness of an image pixel, and $n$ is the number of pixels in the image.

Scaling is performed by expansion and compression of the histogram width (brightness contrasting) by a value determined using the comparison of dispersions or rms deviations by brightness values in the images [15]. Consequently, the compression ratio is determined as

$$
a=\sqrt{\frac{n \sum_{i m g 1} x^{2}-\left(\sum_{i m g 1} x\right)^{2}}{n \sum_{i m g 2} x^{2}-\left(\sum_{i m g 2} x\right)^{2}}},
$$

where $i m g 1$ is the index of the first image, img 2 is the index of the other image, $x$ is the brightness of an image pixel, and $n$ is the number of pixels in the image.

Then, every image pixel changes in accordance with the new brightness value in the histogram scale. This operation allows partial compensation for the distortions occurring at the change in the light intensity.

\subsection{Image Improvement}

The category of the functions improving the images contains speckled background filtering, contrasting, background correction, and additional correction of image defects.

A residual speckled background is present at all the stages of the image input and analysis (Fig. 6a). Therefore, it is eliminated using the known methods of scanning filtering and mathematical morphology. The former is used for reduction of the speckled background and the latter, for shape correction.

However, the use of these methods for magnetooptical image processing is specific. For instance, as the magneto-optical images are characterized by high-density and a high-contrast speckled background, the effectiveness of the "salt \& pepper" method [16] is insufficient. Nevertheless, the use of this filter yields the effect of pixel "granulation" (Fig. 6b). Therefore, 

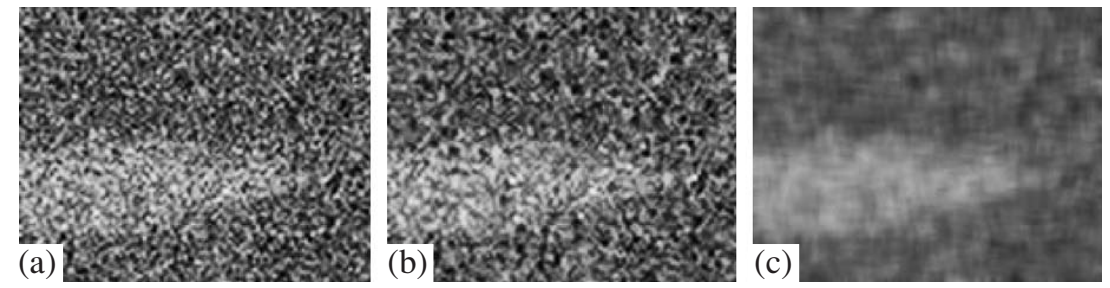

Fig. 6. Speckled background filtering: initial image (a), the image after using a "salt \& pepper" method (b), and the image after lowfrequency averaging filtering (c).
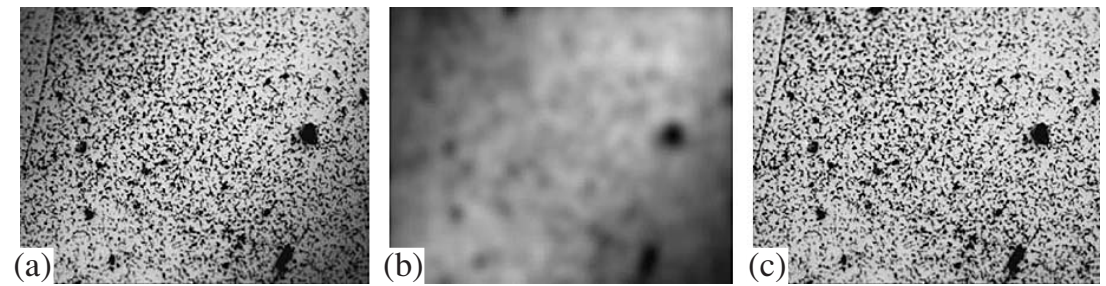

Fig. 7. Initial image: pseudobackground image (a), generated background (b), and the image after background correction (c).
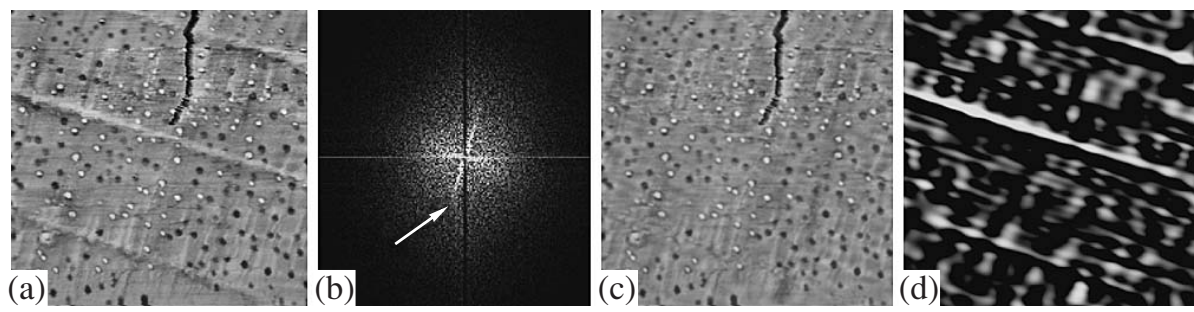

Fig. 8. Frequency features of the image: the initial image where the lines corresponding to the magnetic structure are present (a); the result of the Fourier transformation, where the arrow points to the frequency component of the corresponding line (b), the reconstructed image with the eliminated frequency line (c), and the image reconstructed using the frequency line (d).

we use traditional low-frequency filters (Fig. 6c) for the speckled background reduction.

Brightness distortions are caused by the noise occurring in the optical path during the formation of a sample. According to the possibility of their correction using physical methods, they can be divided into corrected and poorly corrected.

The first category includes the distortions caused by additional light sources (for example, sun light from a window), incorrect microscope tuning, etc. It is better to correct these distortions by tuning the microscope or additional darkening along the optical path, as any mathematical correction allows error.

The second category includes the distortions caused by inclination of the analyzer perpendicular to the axis of rotation at its turning relative to the analyzer (Fig. 7a). In this case, physical correction requires the use of complex high-precision tools and is nearly impossible under normal conditions. For this reason, the use of the image processing is more effective in this case.
The brightness distortion is corrected using the background correction by subtraction of the background image from the initial image. As was shown above, the background image will be different for each angle of rotation of the analyzer. Therefore, these images are inputted and stored for a nonmagnetic sample, forming a bank of additional background images corresponding to different positions of the analyzer.

However, such a correction operation is not always possible, as the refection properties of a nonmagnetic material often strongly differ from those of the magneto-optical sample and, for this reason, one cannot obtain additional background images [11]. In such cases, we propose to use the following procedure for obtaining the background image. The procedure is based on filtering low geometric frequencies [17], which makes it possible to create a pseudobackground image (Fig. 8b) whose difference from the initial image yields the image of object boundaries without optical distortions (Fig. 8c). 


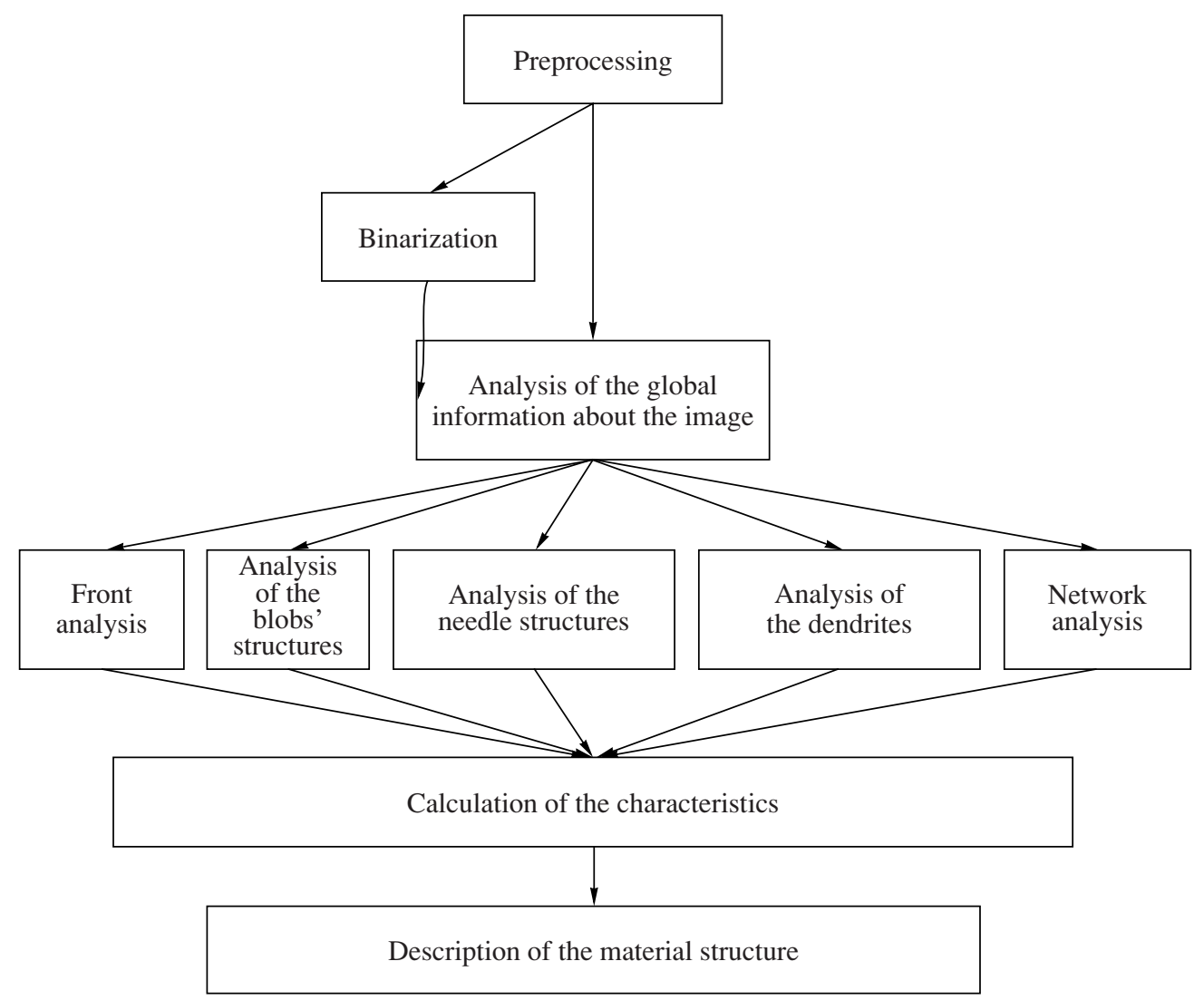

Fig. 9. Analysis of the magneto-optical structure.

Another group of functions for image improvement is transformations. Image transformation is a change in the visual quality of the obtained image. There exist two main reasons for the transformation. First, the improved images are better comprehended by the human eye. This is important for documentation of information and not for the quantitative analysis. Second, the transformation of images changes their characteristics so that, during the quantitative analysis of the images, the objects or phases of interest are best selected. However, it is important to remember that any transformation leads to the loss of some primary information. In any case, one must try to capture the images of the best quality at the input.

The transformations are made using different methods that can be conventionally divided into the following three groups.

1. Changing the image brightness and contrast and smoothing filtering. These transformation methods change the brightness characteristics of an image, which is reflected in the brightness histogram of the image. These functions operate with the brightness histogram.

2. Geometrical transformations. They change the geometrical properties of an image and include rota- tion, size change, and mirror, affine, and projective transformations.

3. Frequency transformations. This group includes the Fourier and wavelet transformations, which allow estimation of the images in terms of geometrical frequencies, determination of the sizes of the most frequently repeating elements in the magnetic structures (Fig. 8), and effective elimination of regular noise occurring in the magneto-optical images.

\section{SELECTION AND ANALYSIS OF THE STRUCTURES}

The analysis of static objects consists of the analysis of arbitrary objects and the analysis of special objects.

The analysis of arbitrary objects requires a specially prepared binary image in which the objects under study are presented by binary images. In the image, one should specify the scale, which can be determined by calibration, and the characteristics to be calculated.

The analysis of special objects requires only preliminary image calibration. This process includes preprocessing, segmentation, shape correction, and determination of the image characteristics. 

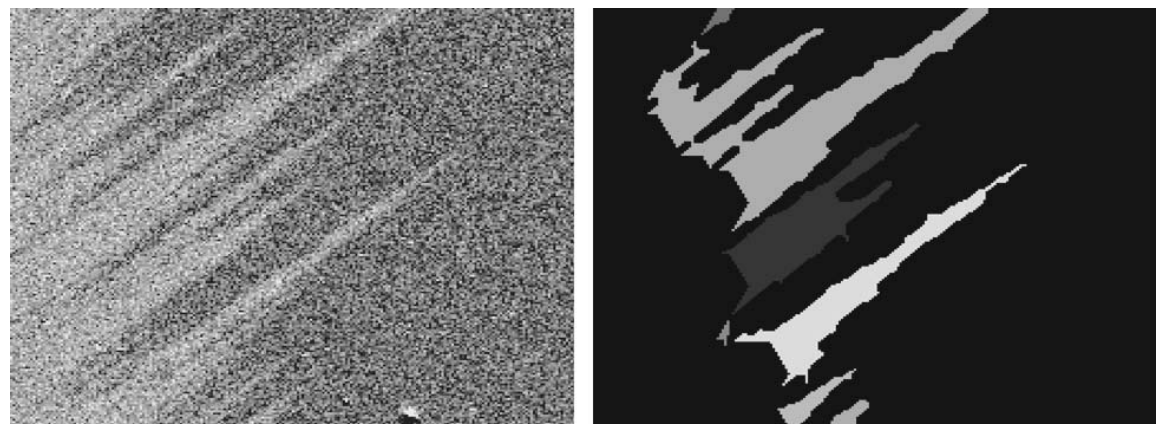

Fig. 10. Results of the direct analysis of needlelike objects.

As was mentioned above, the special objects are divided into four types: blobs (a), fronts (b), needles (c), and dendrites (d).

The blobs and fronts are accompanied by simple algorithms of object selection and calculation of such simple characteristics as the square, length, shape factor, perimeter, etc.

For the needles, two algorithms are proposed: the direct analysis of the objects and the analysis of the objects' boundaries. The first algorithm uses the mathematical morphology operations; it selects the needlelike objects in the image as integral objects and calculates their various characteristics. However, it has several shortcomings. It does not allow for recognition of small needles and merges them with large ones. Another shortcoming is the strong sensitivity to the speckled background. A speckled background grain significantly contributes to the object boundaries, inserting distortions on top; it results in a small error inserted in the orientation of such objects.

The second algorithm does not, in principle, select the object; it is based on tracing the boundaries. The algorithm builds straight lines and ignores small angle distortions. As a result, it is possible to calculate the orientation of the needles more precisely.
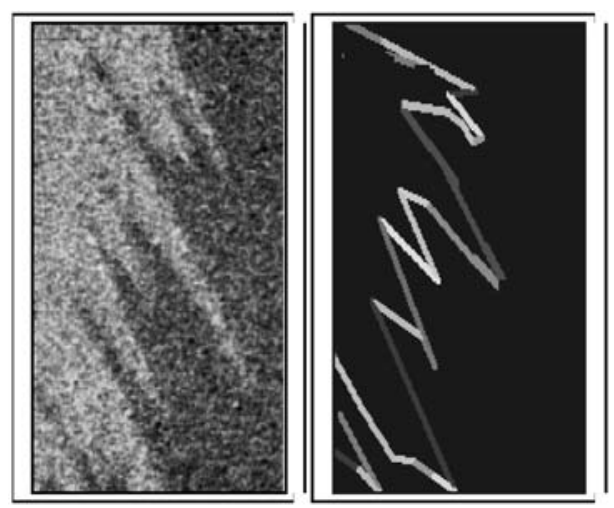

Fig. 11. Results of the analysis of the boundaries of needlelike objects.
The analysis of the needles is accompanied by an additional function of the orientation analysis and building an orientation diagram (asymmetry), which allows judging the dominant needle growth.

The analysis of the dendrite structure uses the most complex algorithm, including mathematical morphology functions and topographical representations. In addition to the traditional geometrical characteristics on the basis of the distance map, it allows calculation of the object width distribution. In terms of the nodes, the branchiness and tailiness, the characteristics of the branches and fingers, and the dendrite complexity are calculated.

\section{ANALYSIS OF THE DYNAMIC CHANGES IN THE MAGNETIC STRUCTURES}

The magnetic structures are not constant objects; they change depending on various physical factors (the

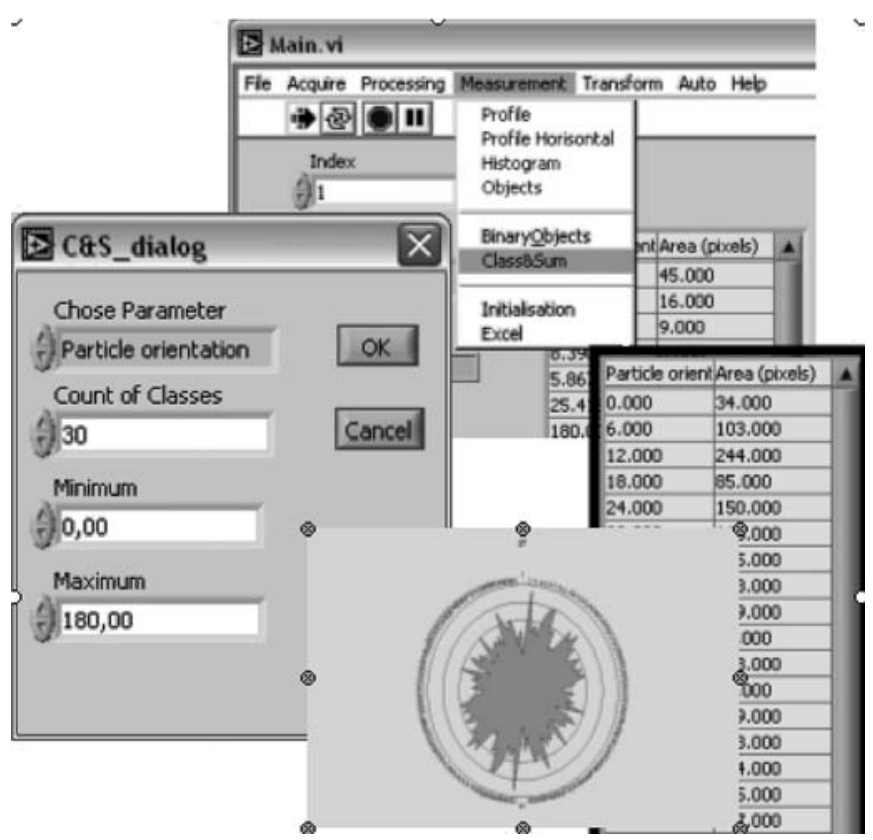

Fig. 12. Fragments of the screen copies with the results of the orientation features and diagram asymmetry. 


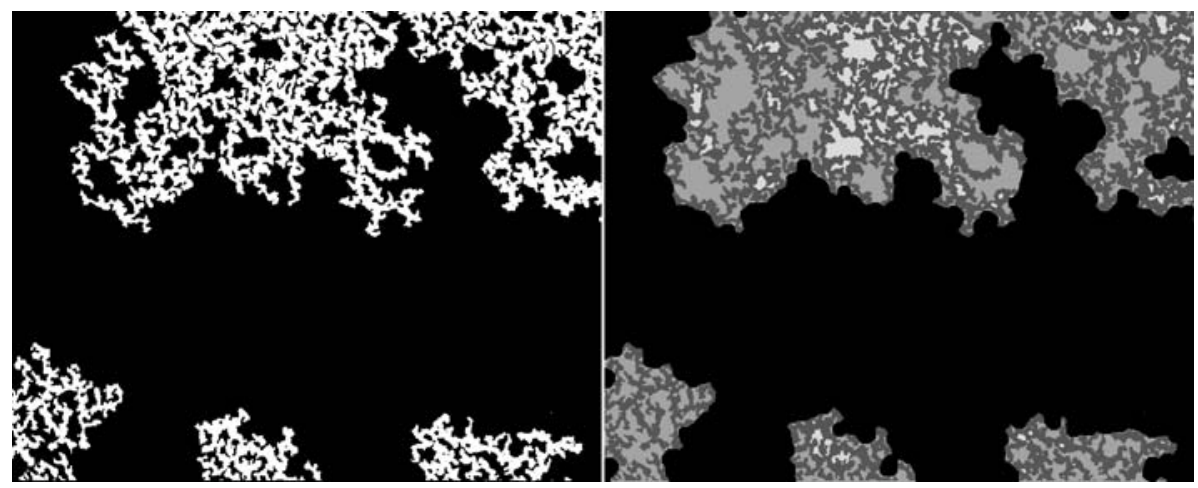

Fig. 13. Result of the analysis of a dendrite object. The dendrite body, gulfs, and holes are shown by different colors.

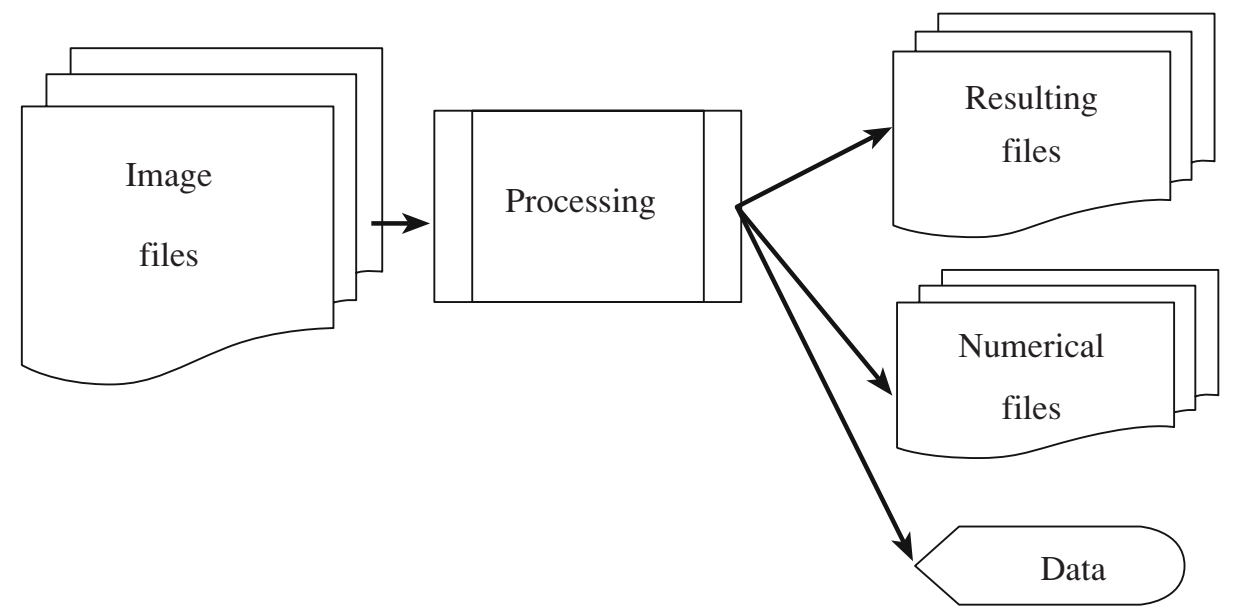

Fig. 14. Scheme of obtaining results using the dynamic analysis functions.

magnetic field, the temperature, the time, the radiation, etc.). Therefore, the investigation of the dynamic changes is of the most interest for a researcher. For this reason, functions of the analysis of the dynamic changes are developed in the software package. These functions operate with a sequence of the images that are recorded in the files whose names contain an image number index. These functions produce a set of binary image files and a file with the dynamic characteristics of the change in the magnetooptical structure.

The dynamic function algorithms double the algorithms of the static analysis functions but allow calculation of the speed and acceleration of the changes in the characteristics.

\section{ZUBR SOFTWARE COMPLEX FOR MAGNETO-OPTICAL IMAGE PROCESSING}

The ZUBR software package is a complex for investigation of magneto-optical images. It includes tools for the interactive and automatic study of one static image and bound sequences of images. However, physical investigation suggests studying the dynamics of a physical process. Therefore, the ZUBR package includes two variants for investigation of the dynamic sequence of images (Fig. 13). The first variant was described above; there exist four main classes of the objects for which the image features are known and the algorithms are developed with the implemented special functions that can be called from the package system menu. The second variant suggests the use of automatic and interactive functions for a static image. In this case, all the actions of a researcher working with the package are stored and recorded in a history text file. Each history operation corresponds to a menu point. In the package, an interpreter is implemented that allows decoding records and repeating the actions of a researcher for another image.

The overlay for operation with the history script is equipped with the required functions and includes a text editor; a binary switch key for storage of the actions of the researcher; a binary switch key for starting the script; and buttons for cleaning the history, recording the history on a disk, and reading from the disc. 


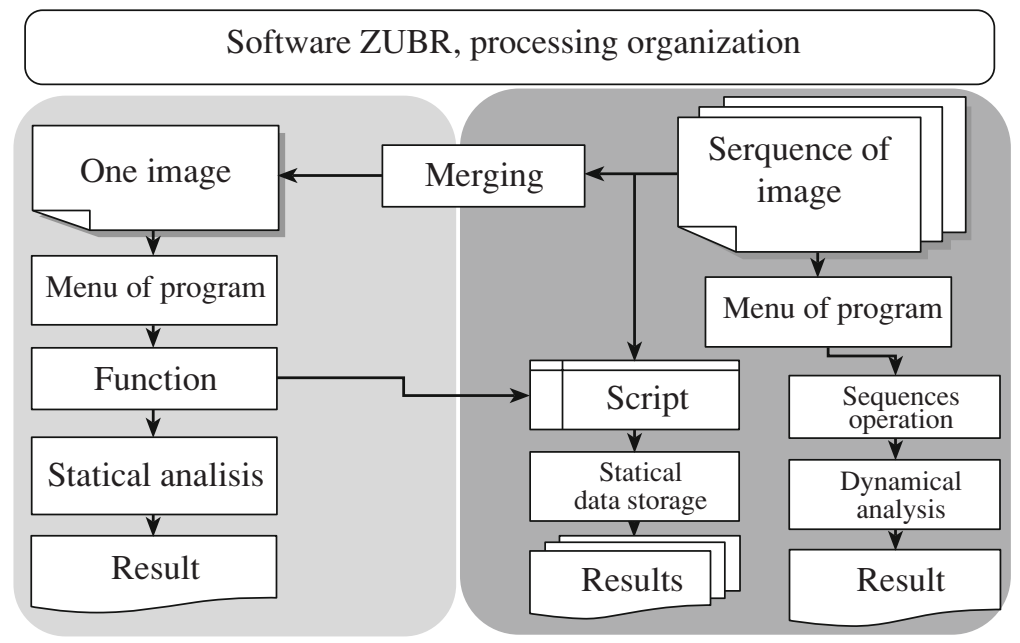

Fig. 15. Organization of the ZUBR software operation with a sequence of images.

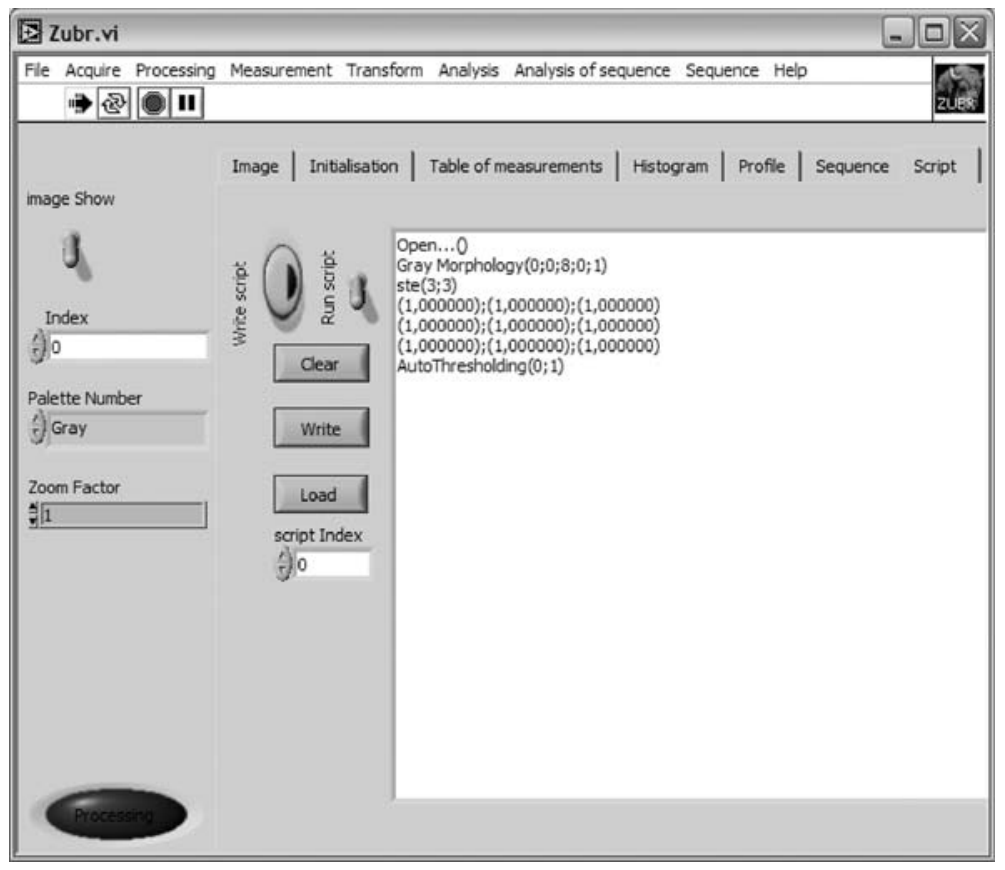

Fig. 16. Copy of the inlay screen for the operation with the history script.

The ZUBR software is divided into three groups (Fig. 17):

1. General functions of image processing and analysis.

2. Analysis of the static magnetic structures.

3. Analysis of the dynamic changes in the magnetic structures using a sequence of images.

The group of general functions of image processing includes traditional methods and algorithms for operation with the images (Fig. 17) and is divided into the following categories:

- Image input and output.
-Image improvement.

- Image segmentation.

- Image transformation.

-Interactive analysis.

The group for the analysis of static images consists of the following categories:

-Measurements of arbitrarily selected objects. The measurement methods are divided by their properties into binary objects, image regions, and calculation of the fractal properties.

-Analysis of blobs.

—Analysis of needles. 


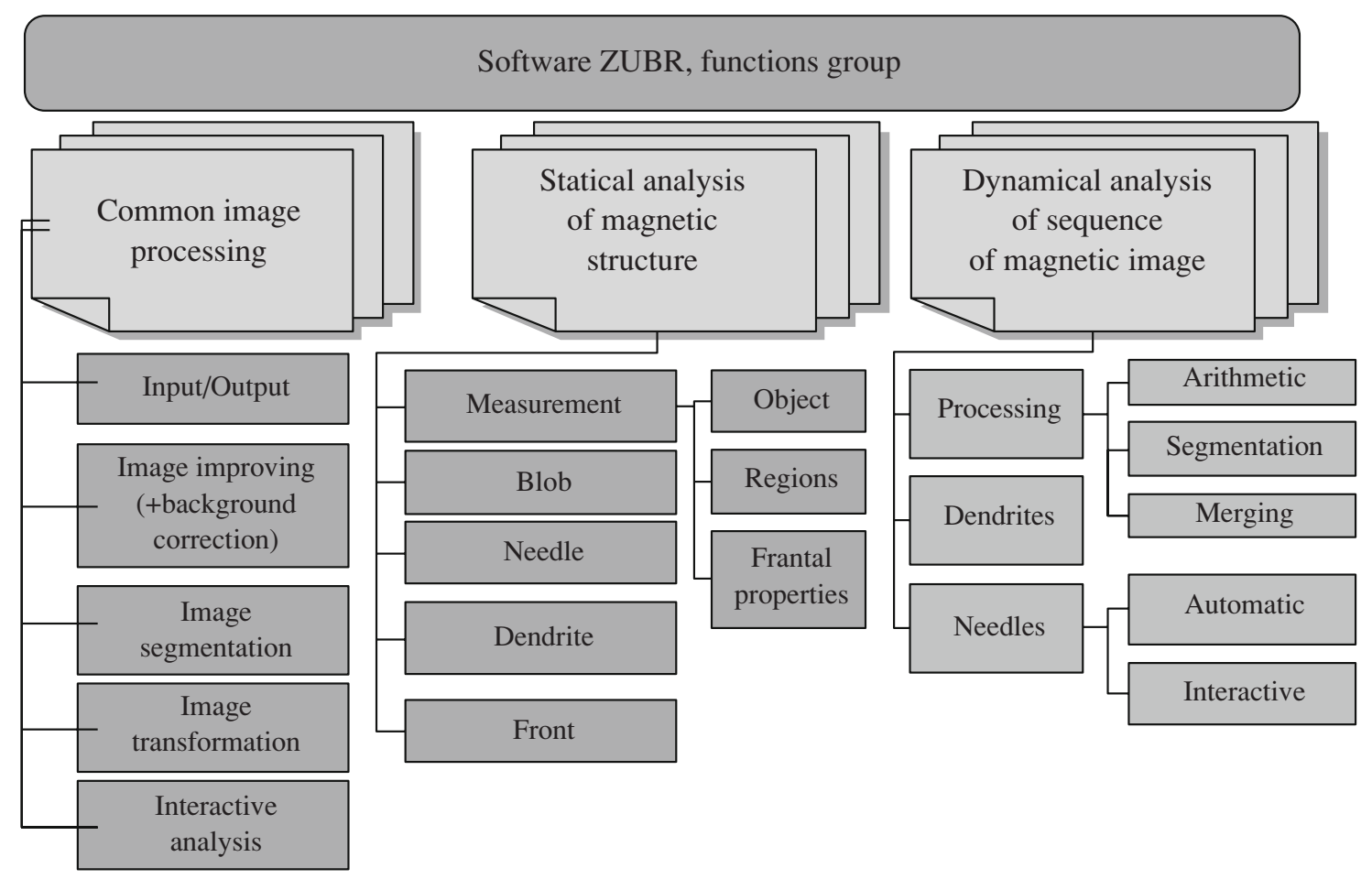

Fig. 17. Functional structure of the ZUBR software.

-Analysis of dendrites.

- Analysis and calculation of the front characteristics.

The analysis includes selection of the algorithms, binarization, correction of the objects, and calculation of their characteristics.

The group of the analysis of the dynamic changes in the magnetic structures functionally doubles the functional for static images but operates with a sequence of images and calculates the dynamic characteristics.

\section{CONCLUSIONS}

The analysis of the magneto-optical images includes two main stages. They are the image preprocessing and selection and the analysis of the structures. The preprocessing is a common stage for all the images, whereas the selection and analysis depend on the structure.

The proposed scheme of preprocessing the magneto-optical images allows partial correction of distortions upon their obtaining due to the instability of the light source, the mechanical shift of the sample, the presence of physical defects, etc. The proposed scheme is generalized. The correction of some defects is not relevant for solving many special problems, as these defects do not influence the result of the investigations. In this case, the stages of defect elimination can be omitted. For instance, the study of the geometrical sizes of small structures does not require the union of images, as the structures do not go beyond the field of view. Here, it is necessary to adjust the proposed preprocessing scheme for solving the specific problem.

The analysis is performed for the basic types of structures that are met in a magneto-optical image. They are blobs, fronts, needles, and dendrites. Both the static images and bound sequence of images are analyzed depending on the changed physical parameter.

This technique is implemented in the ZUBR software complex and has been successfully tested. The package was developed in a LabView shell, which makes it possible to use the possibilities of controlling the tools of the physical experiment. Furthermore, LabView is based on modules of virtual tools, which allows easy using of the materials of the ZUBR software for other purposes.

\section{ACKNOWLEDGMENTS}

This study was supported by the Polish State Committee for Scientific Research (project no. 4 T08A 025 23) and the EU project "Transfer of Knowledge" NANOMAG-LAB (project no. MTKD-CT-2004003177).

\section{REFERENCES}

1. M. Kisielewski, A. Maziewski, M. Tekielak, et al., "New Possibilities for Tuning Ultrathin Cobalt Film Magnetic Properties by a Noble Metals Overlayer," Phys. Rev. Lett. 89 (8), 87203 (2002). 
2. W. Szmaja, "Digital Image Processing System for Magnetic Domain Observation in SEM," J. Magnetism Magnetic Mater. 189 (3), 353 (1998).

3. M. Yukio, I. Hisafumi, and K. Hitoshi, "Automatic Visual Inspection System for Thin Film Magnetic Head Wafer Using Optical Enhancement and Image Processing Technique," in Proc. SPIE Applications of Digital Image Processing $X V$, Ed. by A. G. Tescher (SPIE Homepage) 1771, 20 (1993).

4. Q. Zhu, X. Wang, X. Zou, et al., "Feature Extraction for Magnetic Domain Images of Magneto-Optical Recording Films Using Gradient Feature Segmentation," J. Magnetism Magnetic Mater. 248 (2), 292 (2002).

5. L. Wang, Z. Fan, and D. E. Laughlin, "Trace Analysis for Magnetic Domain Images of L10 Polywinted Structures," Scripta Materiala 47 (11), 781 (2002).

6. D. Sellmyer and R. Skomski, Advanced Magnetic Nanostructures (Springer, 2006).

7. R. Hubert and R. Schafer, Magnetic Domains (Springer, Berlin, 1998).

8. M. Kisielewski, A. Maziewski, Z. Kurant, et al., "Magnetic Ordering in Ultrathin Cobalt Film Covered by a Noble Metals Overlayer," J. Appl. Phys. 93 (10), 7628 (2003).

9. S.-B. Choe, D.-H. Kim, and S.-Ch. Shin, "Domain Reversal Dynamics in Ferromagnetic Thin Films of Co/Pd Nanomultilayers," J. Korean Phys. Soc. 40 (3), 421 (2002).

10. St. Egelkamp and L. Reimer, "Imaging of Magnetic Domains by the Kerr Effect Using a Scanning Optical Microscope," Measur. Sci. Technol. 1, 79 (1990).

11. P. Paturi, LarsenB. Hvolbaek, B. Jacobsen, and N. Andersen, "Image Correction in Magneto-Optical Microscopy," Rev. Sci. Instr. 74 (6), 2999 (1990).

12. A. Nedzved, S. Ablameyko, M. Tekielak, et al., Processing of Needkes-Like Images of Magnetic Domain Structures in Ultrathin Cobalt Wedge, 8th International Conf. on Pattern Recognition and Information Processing (PRIP'2005), May 18-20, 2005, Belarus, Minsk (2005), pp. 108-111.

13. R. Szeliski, Image Alignment and Stitching: A Tutorial. Technical Report MSR-TR-2004-92 (Microsoft Research, 2005).

14. S. Ablameyko and A. Nedzved, Optical Image Processing of Cells Structure in Medicine (NANB UIIP, Minsk, 2005), p. 155.

15. A. Tinku, K. Ajoy, and I. Ray, Image Processing: Principles and Applications (Wiley, 2005), p. 428.

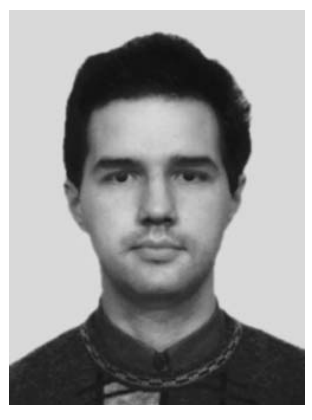

Aleksandr Nedzved graduated from Belarus State University in 1992 and received a candidate's degree in 2000 . He is deputy head of laboratory of image analysis and procesing in United Institute of Informatics Problems of the National Academy of Sciences of Belarus. His scientific interests include the image analysis, object segmentation and morphometry investigation. $\mathrm{He}$ is the author of more than 50 papers and a member of the Belarus Association for Image Analysis and Recognition.
16. G. X. Ritter and J. N. Wilson, Handbook of Computer Vision Algorithms in Image Algebra (CRC, Boca Raton, FL, 1996).

17. S. T. Bow, Pattern Recognition and Image Preprocessing (New York, Basel, 2002), p. 698.

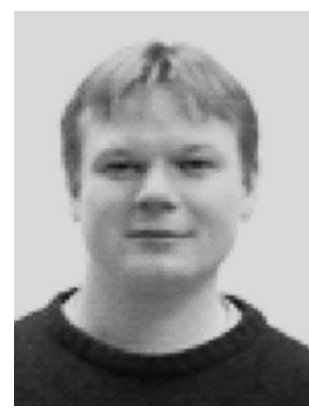

Wojtek Dobrogowski works at the University of Bialystok, and his scientific interests include the computer supported study of magnetic nanostructures and education.

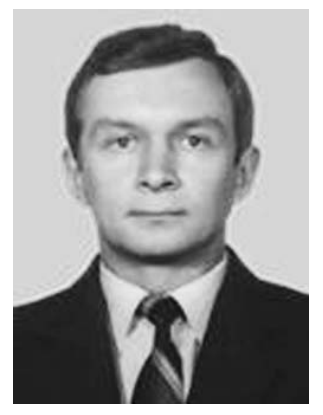

Sergei Ablameyko graduated from Belarus State University in 1978 and received his candidate's degree in 1984 and his doctoral degree in 1990. $\mathrm{He}$ is the rector of Belarusian State University, a professor of the University of Informatics and Radio Electronics, and an advisory editor of the International Journal of Machine Graphics and Vision. His scientific interests include document image analysis, image processing, object representation and recognition, digital geometry, and geographic information systems. He is the author of more than 300 papers, a senior member of the Institute of Electrical and Electronics Engineers, chairman of the Belarus IEEE Center, a member of the Governing Board of the International Association for Pattern Recognition, and 1st Vise-President of International Association for Pattern Recognition Corresponding member of the National Academy of Sciences of Belarus.

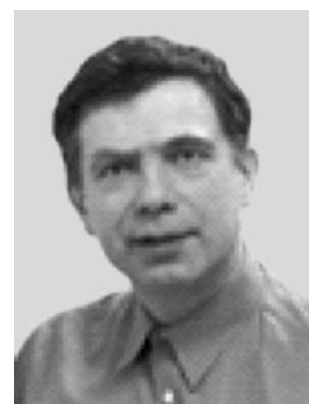

Andrzei Maziewski works at the University of Bialystok, and his scientific interests include magnetic nanostructures and popularization of science.

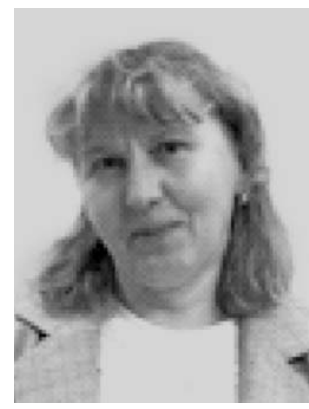

Maria Tekielak works at the University of Bialystok, and her scientific interests include image processing for optical microscopy for ultrthin magnetic films and domain structures analysis, magnetic nanostructures analysis, and popularization of science. 